\title{
INCORPORATION OF UNRELIABLE INFORMATION INTO PHOTOGRAMMETRIC RECONSTRUCTION FOR RECOVERY OF SCALE USING NON-PARAMETRIC BELIEF PROPAGATION
}

\author{
J. Hollick ${ }^{\mathrm{a}, *}$ P. Helmholz ${ }^{\mathrm{a}}$, D. Belton ${ }^{\mathrm{a}}$ \\ a Department of Spatial Sciences, Curtin University, Australia - j.hollick@ postgrad.curtin.edu.au, (petra.helmholz, d.belton)@curtin.edu.au
}

\author{
Commission V, WG V/4
}

KEY WORDS: Sensor Fusion, Belief Propagation, Photogrammetry, Barometer, GNSS

\begin{abstract}
:
The creation of large photogrammetric models often encounter several difficulties in regards to geometric accuracy, scale and geolocation, especially when not using control points. Geometric accuracy can be a problem when encountering repetitive features, scale and geolocation can be challenging in GNSS denied or difficult to reach environments. Despite these challenges scale and location are often highly desirable even if only approximate, especially when the error bounds are known. Using non-parametric belief propagation we propose a method of fusing different sensor types to allow robust creation of scaled models without control points. Using this technique we scale models using only the sensor data sometimes to within $4 \%$ of their actual size even in the presence of poor GNSS coverage.
\end{abstract}

\section{INTRODUCTION}

As the number and quality of sensors increases the need to have a method of fusing this information also increases. There is currently a significant amount of research into fusing multiple different sensors, each for different purposes, these include reliability (Lhuillier, 2012) and reducing drift (Kerl et al., 2013). This research has resulted in several different forms of sensor fusion each with its own limitations. For instance Bayesian based methods are popular, however need to have the error distributions of the sensors specified a priori (Koks and Challa, 2003). DempsterShaefer based methods do not require this information however struggle to deal with conflicting measurements from sensors (Ali et al., 2012), (Jøsang and Pope, 2012), (Klein, 1993).

The number of sensors available at a reasonable cost to the consumer is also increasing, for example the number of sensors on smart phones increasing in number and quality each year. However, when compared to sensors specifically designed for navigation their accuracy is lacking. Despite their lower accuracy these sensors still find uses in many applications such as orientation finding (Ayub et al., 2012), activity identification (Tundo et al., 2013) and geotagging for Structure from Motion (Crandall et al., 2013).

In this paper we present a method of fusing accelerometer, barometric and GNSS measurements along with images using a nonparametric belief propagation and photogrammetry. We show preliminary results with real-world data from a commodity smart phone and digital camera. In section 2 we outline belief propagation in both the discrete and non-parametric forms. We then describe our method in section 3 followed by some initial results and comparisons in section 4. Finally, our conclusions and outline of intended future work is presented in section 5 .

\section{BELIEF PROPAGATION}

\subsection{Discrete Belief Propagation}

Belief Propagation over discrete variables was first published by Judea Pearl in 1982 as an efficient method of solving the marginal

\footnotetext{
${ }^{*}$ Corresponding author
}

probabilities inside Bayesian Tree graphs (Pearl, 1982). Since then it has proven useful on the more general cyclic graphs. However, the conditions for convergence on these graphs, if indeed it does converge, are not well understood at this point (Murphy et al., 1999), (Yedidia et al., 2000) and (Yedidia et al., 2003). Despite this, in many cases the resulting approximations are still useful (Murphy et al., 1999), (Ihler et al., 2005).

Belief propagation also has the advantage that the state of each node is dependant only on the messages coming into it. This means that once the network stabilises, if new data is inserted or a change is made. The change will flow through the network and only those nodes that receive a new and different message will change their state. Depending on the network this may mean that a significant number of the nodes do not need to redo their calculations.

One of the most important parts of belief propagation are the conditional probability functions linking the various nodes in the graph (Pearl, 1982), (Yedidia et al., 2003) as these functions define the computation that will take place.

The following steps outline the general belief propagation algorithm (Yedidia et al., 2003):

1. Define graph structure and compatibility functions

2. Set variables where known to their desired states.

3. During each iteration node $i$ sends message $M_{i, j}$ to neighbour $j$. Where $M_{i, j}$ contains a set of states and the probabilities that node $j$ will be in those states according to the information available at node $i$. Excluding the message from node $j$.

4. Step 3 is repeated until either the messages no longer change or the number of allowed iterations has been reached

The messages passed between nodes are calculated using equation 1 below:

$$
M_{i, j}=\sum_{x_{i}} \phi\left(x_{i}\right) \psi_{i j}\left(x_{i}, x_{j}\right) \prod_{\forall k \in N(i) \backslash j} M_{k i}
$$


Where $M_{i j}$ is the message from node $i$ to $j, \phi\left(x_{i}\right)$ is the evidence at node $i$ and $\psi\left(x_{i}, x_{j}\right)$ is the compatibility function between nodes $i$ and $j$. In the case of discrete belief propagation this is a vector with as many elements as possible states.

When the algorithm described above has completed the final states of the nodes is calculated using the following equation:

$$
b_{i}=k \phi\left(x_{i}\right) \prod_{\forall k \in N(i)} M_{k i}
$$

where $\phi$ and $M_{i j}$ are defined as above and $k$ is a constant such that $\left|b_{i}\right|=1$.

\subsection{Non-Parametric Belief Propagation}

Belief propagation was initially described for discrete states, however for many complex systems discretising the variables of interest is either infeasible or impossible. Sudderth et al. (2003) proposed a non-parametric form of belief propagation that uses Gaussian Mixture Models (GMMs) to represent the variables of interest. This avoids the problems associated with discretising the variables and allows any state space to be used.

This non-parametric form retains many of the benefits of the discrete belief propagation the only significant difference is that the variables are represented by probability distributions. These distributions can be of any form that allows operations to be performed on them. One limitation of this technique is that the number of kernels in each of the GMMs increases as a result of many operations. This has computational implications as the more kernels there are the longer the computation will take. In order to reduce this problem, Sudderth et al. (2003) propose to use Gibbs sampling to reduce the number of kernels in a GMM. This effectively trades computation time for accuracy and a balance is required.

\section{METHOD}

\subsection{Coordinate Systems}

The process of fusing the different sensors requires that we maintain a common coordinate system or transform between different systems suitable for each sensor so that we can define relationships between them. We use the right handed East-North-Up (ENU) coordinate system. This has several advantages including the natural separation of height and position. This also allows us the freedom to use either a local or globally referenced coordinate system as the only difference between them is the georeferencing. This is also the same coordinate system as the Android environment (Google, 2016b) which simplifies our modelling as extra transforms are not required.

When global positioning such as GNSS is used, we use the first valid coordinate as the origin and build up from there. In the case of barometric data we can lock the initial heigh to zero or set one of the known heights without requiring the other axis to be set.

We store all our orientations as $(\theta, \phi, \rho)$ where $\theta, \phi$ encode 'down' and $\rho$ is the heading. We also limit each of $\theta, \phi, \rho$ to $(0, \pi)$, $(0,2 \pi)$ and $(0,2 \pi)$ respectively. We can then compute the rotation from a local coordinate system to a global system as shown in equation 6

$$
R_{y}(\theta)=\left[\begin{array}{ccc}
\cos (\theta+\pi) & 0 & -\sin (\theta+\pi) \\
0 & 1 & 0 \\
\sin (\theta+\pi) & 0 & \cos (\theta+\pi)
\end{array}\right]
$$

$$
\begin{aligned}
& R_{z}(\phi)=\left[\begin{array}{ccc}
\cos (\phi) & \sin (\phi) & 0 \\
-\sin (\phi) & \cos (\phi) & 0 \\
0 & 0 & 1
\end{array}\right] \\
& R_{z}(\rho)=\left[\begin{array}{ccc}
\cos (\rho) & \sin (\rho) & 0 \\
-\sin (\rho) & \cos (\rho) & 0 \\
0 & 0 & 1
\end{array}\right]
\end{aligned}
$$

combining to give a rotation of

$$
R(\theta, \phi, \rho)=R_{z}(\rho) R_{y}(\theta) R_{z}(\phi)
$$

where

$$
\begin{aligned}
& \theta \text { is the latitude where } 0 \text { is }+z \text { and } \pi \text { is }-z \\
& \phi \text { is longitude such that when } \theta=\pi / 2,0 \text { is }+x \\
& \text { and } \pi / 2 \text { is }+y \\
& \rho \text { is the heading clockwise from north }
\end{aligned}
$$

Using this system of coordinates we can independently calculate up and the heading which allows certain useful cases. For example, with accelerometer data we can easily calculate the direction of gravity, whereas the heading cannot be determined. Once global coordinates are introduced the heading can be determined without changing our coordinate system.

\subsection{Sensor Models}

The measurements from a barometer can be used in two ways, firstly to calculate an absolute height and secondly to calculate the relative height difference between two points. Currently in our work we do not attempt to calculate the absolute height as this would require a reference pressure. This reference would change over time making the calculation unreliable unless this reference is constantly updated. Instead we use the barometer to calculate relative heights between successive measurements. The calculation for relative height difference is given in equation 7 below (Engineering Toolbox, 2016):

$$
h_{2}-h_{1}=\frac{\exp \left(\frac{\ln \left(\frac{P_{1}}{C_{1}}\right)}{C_{3}}\right)-\exp \left(\frac{\ln \left(\frac{P_{2}}{C_{1}}\right)}{C_{3}}\right)}{C_{2}}
$$

where $\quad P_{1}, P_{2}=$ Pressure in $\mathrm{Pa}$ at points one and two

$h_{1}, h_{2}=$ Heights at points one and two

$C_{1}=101,325$

$C_{2}=2.25577 \times 10^{-5}$

$C_{3}=5.25588$

When taking measurements from the accelerometer we generate an estimate of 'down', allowing us to estimate the orientation of the sensor platform at each measurement using the following equations:

$$
\begin{gathered}
\theta=\sin ^{-1}\left(\frac{A_{z}}{\left|\left[A_{x}, A_{y}, A_{z}\right]\right|}\right)+\frac{\pi}{2} \\
\phi=\cos ^{-1}\left(\frac{-A_{x}}{\left|\left[A_{x}, A_{y}\right]\right|}\right)
\end{gathered}
$$

where $\quad A_{x}, A_{y}, A_{z}$ are the accelerometer measurements $\theta, \phi$ are defined as above. 
In order to resolve the ambiguity of $\phi$ when $A_{x}>0$ we set

$$
\phi=2 \pi-\phi
$$

While we model many primitive sensors there are some sensor platforms such as digital cameras which are a combination of sensors and as such we construct the sensor platforms from a combination of our primitive sensors. A modern digital camera records many more details that just image data, other common data may include: focal length, timestamp, geolocation from GNSS and orientation. We intend to use all of these details in future versions of our fusion algorithm. When using a smart phone to log different sensors we take the latest value from the available sensors and tag the image with those values.

\subsection{Sensor Calibration}

In our work we assume that sensor measurements from the accelerometer and barometer can be calibrated by using a scale and offset as shown in equation 11 below and their errors modelled with a Gaussian distribution. In the case of GNSS these error distributions are provided directly by the sensors otherwise are the result of calibrations.

$$
X_{c}=X_{u} \cdot M+O
$$

where $\quad X_{u}$ are the uncalibrated measurements

$M$ are the scales for each axis

$O$ are the offsets after scaling

$X_{c}$ are the calibrated measurements

Accelerometers and barometers generally do not record their uncertainties, so we are required to perform a calibration in order to specify the uncertainties in our model. We then assume that this calibration is stable and holds for the whole data collection period.

For an accelerometer this calibration is performed by recording a series of measurements while the device is stationary in several different orientations. We then solve equation 11 such that $\left|X_{c}\right|=9.81 \mathrm{~ms}^{-1}$ in order to find the scale and offset values for each axis. We also assume that the axis of the sensors are orthogonal, again negating the need for more complex calibrations.

For GNSS sensors we use the accuracy reported by the GNSS system to provide the variance of the Gaussian error distribution. On Android systems the error reported by the GNSS system is the standard deviation of a Gaussian distribution which fits our model (Google, 2016a).

Calibration of the barometer has been performed by recording barometer measurements for 5 minutes then computing the standard deviation of the results. At this stage calibration for possible scale error is not performed as for the heights and time periods in current testing this is unlikely to be significant.

For barometers there is another step that should be performed at the time the measurements are taken for greater accuracy. In many places for the scales we are looking at the local air pressure changes significantly even in short periods of time. To combat this we record the air pressure at the beginning and end of the capture period from the same point and calculate a correction factor using equation 12 below. This increases accuracy significantly.

$$
\operatorname{correction}(\tau)=\tau \frac{P_{2}-P_{1}}{t_{2}-t_{1}}
$$

In our calibrations at this stage we assume that the offset between the different sensors is negligible, allowing us to omit several variables from our model. However, with more accurate sensors or larger distances between them this is something we would have to take into account.

\subsection{Belief Propagation Algorithm}

A non-parametric belief propagation algorithm is implemented following similar principles to Sudderth et al. (2003) and Crandal et al. (2013). Each of our variables is represented as a bounded GMM with a uniform component as described in equation 13:

$$
\left\{\mu_{i}, \sigma^{2}, w_{i}\right\}_{i=1}^{K}, u,\left\{\lambda_{\text {lower }}, \lambda_{\text {upper }}\right\}
$$

where $\quad\left\{\mu_{i}, \sigma^{2}, w_{i}\right\}$ are the mean, variance and weight of the $i^{\text {th }}$ kernel in the GMM $u$ is the uniform distribution $\left\{\lambda_{\text {lower }}, \lambda_{\text {upper }}\right\}$ are the upper and lower bounds

The use of a uniform distribution is not universal, it is not described by Sudderth et al. (2003), however Crandall et al. (2013) find that it assists with convergence when conflicting messages are present.

Many operations on GMMs increase the number of elements in the result. Which results in increased computation time. To combat this resampling methods are often used. Sudderth et al. (2003) use Gibbs sampling, whereas we use a more simple hill climbing method. We use the means of the kernels as a potential starting point. Firstly, selecting the mean with the largest value of the GMM at that point then using Newton-Raphson to refine. Using this value we calculate the variance and weight then create a new distribution in the new resulting sub-sampled GMM. This algorithm is detailed below:

1. Find the maximum of the current GMM by testing each of the internal distributions and the upper and lower bounds then use up to 10 iterations of Newton-Raphson to refine to local maximum saving the best $\mu$ and $v$. Where $\mu$ is the position of the maximum and $v_{\max }$ is the value.

2. If $v<$ threshold then end.

3. Set $\sigma^{2}$ to the average kernel variance in the GMM.

4. Set varStep $=\sigma^{2} / 2$

5. Calc $w=v_{\max } \sqrt{2 \pi \sigma^{2}}$

6. $e r r=f\left(x \mid \mu, \sigma^{2}\right)-G M M\left(\mu+\sigma^{2}\right)-G M M\left(\mu-\sigma^{2}\right)$

7. If $\mid$ err $\mid<$ allowErr then goto 11

8. If $\mathrm{err}>0$
(a) then $\sigma^{2}+=$ varStep
(b) else $\sigma^{2}-=$ varStep

9. Set varStep/ $=2$

10. Goto 5

11. Add new kernel to current GMM with $\left\{\mu, \sigma^{2},-w\right\}$ and new kernel to new GMM with $\left\{\mu, \sigma^{2}, w\right\}$.

12. Repeat from 1 until $K$ kernels are in the new GMM.

Where $f\left(x \mid \mu, \sigma^{2}\right)$ is the evaluation of an individual Gaussian distribution with mean and variance $\sigma^{2}$. GMM $(x)$ is the evaluation of a Gaussian Mixture Model at $x$. Once this algorithm has 
completed we combine the remaining probabilities into the uniform distribution of the new GMM. Afterwards, we normalise the weights so that the sum of all weights and the uniform distribution equals one.

This resampling is run after the calculation of message values so that the messages are both normalised and not 'too large'. In our implementation we have chosen $K=20$, allowErr $=0.01$ and threshold $=0.001$. These values are chosen as a compromise between speed and accuracy, larger values of $K$ and lower values of allowErr and threshold are more accurate but slower.

\subsection{Belief Propagation Nodes}

We define three different types of nodes in our implementation of belief propagation, these are constant, function and variable nodes. The constant and variable nodes are essentially the same in that they do not perform any significant processing and only maintain a value. The difference is that the constant nodes have their variable set on initialisation and thereafter it does not change whereas the value of the variable nodes changes in response to incoming messages.

The function nodes on the other hand implement various processing steps and in many cases also store a value. This is done to simplify the resulting graph that is created. While it would be possible to create a graph from only variable and function nodes that do not have state it would be much larger and more complex.

Our barometer nodes are functional nodes that combine both a sensor measurement and a functional node that calculates the relative height change between connected nodes. Each barometer node passes its estimated height and local pressure measurement to the nodes around it. This allows the neighbouring barometer node to estimate the height difference using the pressure difference and equation 7 hence calculating its local height. This is then combined with other height estimates passed to this node to give an estimate of the height at this node.

Our accelerometer node uses equations 8 and 9 internally to estimate its own orientation which is then passed as part of the outgoing message along with the acceleration values measured.

We implement our image nodes as simple variable nodes that do not do any processing but just combine position and orientation estimate from connected nodes.

The last node required for this implementation is an 'Image Match' function node. This node contains the relative orientation between two images as calculated using photogrammetry. In a similar way to the barometer node outlined above this node takes the orientation of one image then provides an estimate of the orientation of the other connected image. At this point this relative orientation is precalculated using Agisoft Photoscan (Agisoft, LLC, 2016).

\subsection{Graph Generation}

Using the nodes described above we can automatically generate a graph from a dataset of sensor measurements at run-time. At this stage we create an image node for each image and constant or function nodes from the sensor measurements as described above. These constant nodes apply the calibrations and coordinate transforms described above and cache the results internally so they are not recomputed at each iteration step. We then take the relative orientation of the images from another piece of software and create a set of 'Image Match' nodes linking the images. This results in a graph similar to Figure 1 below.

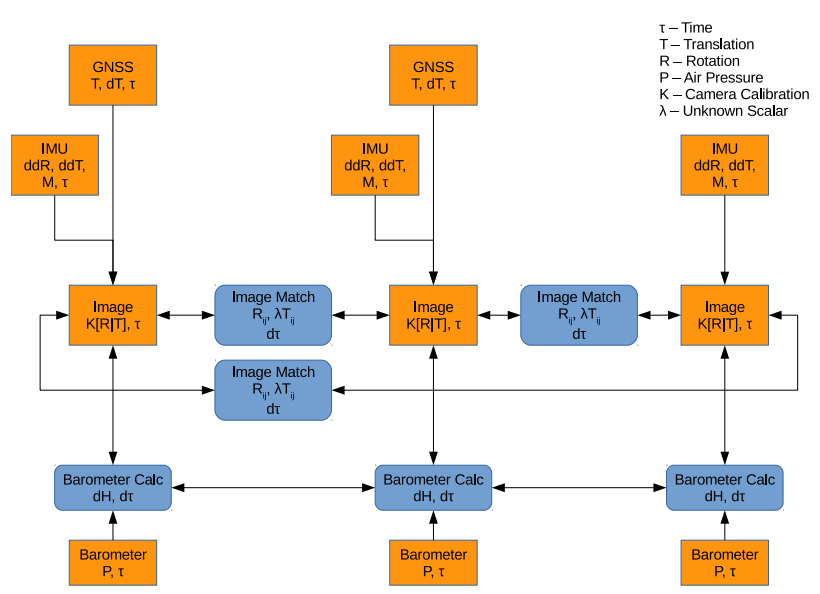

Figure 1: Illustration of a section of a graph generated using our technique. Function nodes in blue and sensor measurement nodes in orange.

\subsection{Scale}

In order to generate a scaled and oriented point cloud we first take a set of images of the object or scene of interest with one or more sensor platforms while logging the relevant sensor information. The process is then as follows:

1. Use Photoscan to generate a point cloud and set of camera positions and orientations.

2. Run our belief propagation algorithm as defined above on the sensor data to generate a geolocation, orientation and position estimates.

3. Take the most likely value for the height and orientation of each camera

4. Rotate Photoscan point cloud using the generated rotation

5. Use least squares to translate and scale the point cloud

The coordinate system used by Photoscan is a right handed coordinate system where the local coordinates for each image are such that $x-y-z$ is down-right-backwards. To transform this into our coordinate system we apply the following transform. Firstly, we apply the inverse rotation of the first camera, then we transform to the coordinate system used by Android so that the coordinates from Photoscan match the coordinates from the Belief Propagation. Finally, we apply our calculated rotation of the first camera so that everything is in our ENU world coordinate system.

$$
\tilde{X}_{E N U}=R(\theta, \phi, \rho)\left[\begin{array}{ccc}
0 & 1 & 0 \\
-1 & 0 & 0 \\
0 & 0 & 1
\end{array}\right] R_{0}^{-1} \tilde{X}_{P S}
$$

$$
\begin{array}{ll}
\text { where } & R_{0} \text { is the rotation of the first camera } \\
\tilde{X}_{P S} \text { a camera location from Photoscan } \\
\tilde{X}_{E N U} \text { a camera location in our ENU system }
\end{array}
$$

We then use least squares to resolve the scale of the final model by minimising the difference between the relative heights as calculated by belief propagation and the relative scaled relative heights from the oriented Photoscan point cloud. 


\section{RESULTS}

\subsection{Sensors}

Real data from a Galaxy SIII smart phone and a Canon 6D digital camera have been used to evaluate our approach. The Galaxy SIII contains a GNSS sensor which reports at approximately $1 \mathrm{~Hz}$ using both GPS and GLONASS. It also contains an accelerometer and barometer which report at approximately $100 \mathrm{~Hz}$ and $25 \mathrm{~Hz}$ respectively. We also use the built in camera to provide image data with the camera fully zoomed out. Under the Android operating system the exact reporting rate is not guaranteed, however timestamps in nanoseconds are given for each of the measurements. Images are not timestamped like the other sensor measurements, so we place a tag in the log file when an image capture was triggered. Then, we assign the image the same timestamp as the previous sensor measurement. The images captured are 8 megapixels (3264×2448 pixels). The Canon $6 \mathrm{D}$ records 20 megapixel (5472x3648 pixels) photographs using a $50 \mathrm{~mm}$ prime lens.

\subsection{Calibrations}

Using the calibration methods described in section 3.3 above, our calibration values for the Galaxy SIII sensors are given in Tables 1 and 2. The error reported by the GNSS system for each measurement was used for the standard deviation. The average standard deviation for each sensor is recorded in Table 3 below.

\begin{tabular}{|l|c|c|c|}
\hline Parameter & $M_{x}$ & $M_{y}$ & $M_{z}$ \\
\hline Accelerometer & 1.0023 & 0.9904 & 0.9888 \\
Barometer & - & - & 1.0000 \\
\hline
\end{tabular}

Table 1: Calibration scales for sensors.

\begin{tabular}{|l|c|c|c|}
\hline Parameter & $O_{x}$ & $O_{y}$ & $O_{z}$ \\
\hline Accelerometer & 0.1149 & 0.0889 & 0.5064 \\
Barometer & - & - & 0 \\
\hline
\end{tabular}

Table 2: Calibration offsets for sensors.

\begin{tabular}{|l|c|c|c|}
\hline Sensor & $\sigma_{x}$ & $\sigma_{y}$ & $\sigma_{z}$ \\
\hline Accelerometer & 0.0206 & 0.0198 & 0.0298 \\
Barometer & & & 7.5400 \\
GNSS - Limestone Blocks & & & 199.89 \\
GNSS - War Memorial & & & 442.07 \\
\hline
\end{tabular}

Table 3: Sensor standard deviations after calibration

To give an idea of scale, a standard deviation for the barometer of $7.54 \mathrm{~Pa}$ in Table 3 results in approximately $0.63 \mathrm{~m}$ of elevation at the standard atmospheric pressure of $100 \mathrm{kPa}$.

\subsection{Datasets}

We have collected data from two areas. Figures 2 and 3 show an example image of each dataset with measurements. For the Limestone Block dataset 35 images from the 6D and 13 images from the Galaxy SIII were captured. For the War Memorial, 36 images from the 6D and 17 images from the Galaxy SIII were captured. These provided enough coverage to create a mesh of the relevant parts in Photoscan.

Neither of these datasets has full GNSS coverage with the Galaxy SIII. Only 8 of the 13 images from the Limestone Block dataset and 15 of the 17 images from the War Memorial dataset have GNSS measurements. In addition, the very large average standard deviations shown in Table 3 for the GNSS suggest that the GNSS data is rather poor.

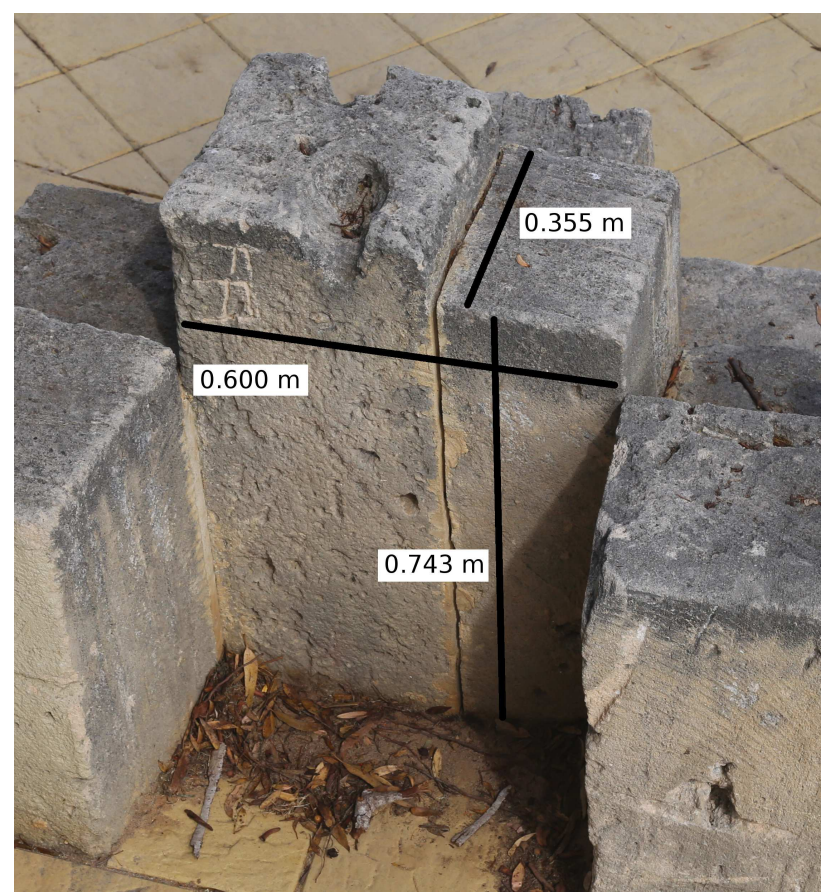

Figure 2: Illustration of the three measurements made on the war memorial dataset. Measurements numbered one to three from top to bottom

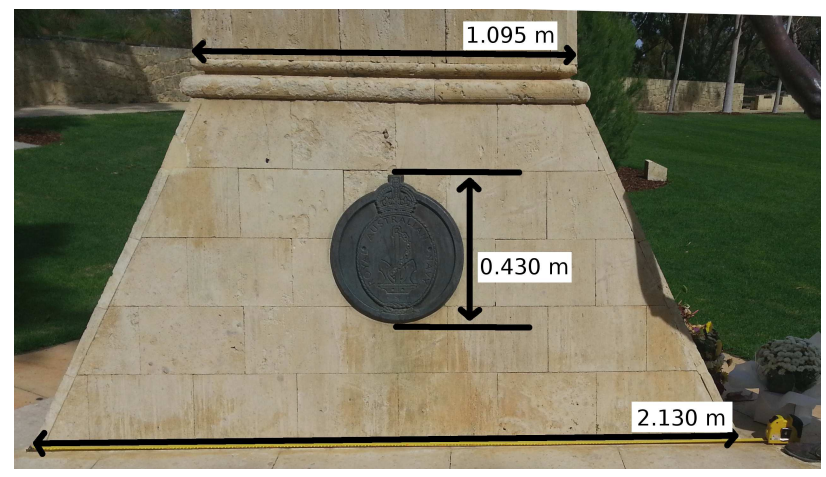

Figure 3: Illustration of the three measurements made on the war memorial dataset. Measurements numbered one to three from top to bottom.

\subsection{Accuracy}

Tables 4 and 5 show the resulting measurements shown in Figures 2 and 3 after the point cloud and mesh have been scaled using the procedure in 3.7.

It is clear that using GNSS alone in this case is near useless as there is just too much error. The scales calculated for these results were 0.000 and -0.020 respectively, hence the nonsense results. However, the barometric data by itself is reasonably good and in the case of the War Memorial dataset the combination of barometric and GNSS measurements proves to be quite accurate especially given the errors of the input data.

Using the results shown in Tables 4 and 5 we calculate the percentage error of each dataset (Table 6). Here it is clear that GNSS measurements do not always increase the accuracy of the results. In the case of the Limestone Blocks the GNSS measurements actually make the final result worse. This suggests that a method 
of excluding truly bad data needs to be implemented in order to resolve this problem.

\begin{tabular}{|l|c|c|c|c|}
\hline Measurement & Actual & GNSS & Barometer & Both \\
\hline 1 & 0.355 & 0.000 & 0.290 & 0.251 \\
2 & 0.600 & 0.000 & 0.502 & 0.435 \\
3 & 0.743 & 0.000 & 0.603 & 0.522 \\
\hline
\end{tabular}

Table 4: Measurements for the Blocks

\begin{tabular}{|l|c|c|c|c|}
\hline Measurement & Actual & GNSS & Barometer & Both \\
\hline 1 & 1.095 & -0.024 & 1.201 & 1.064 \\
2 & 0.430 & -0.009 & 0.444 & 0.394 \\
3 & 2.130 & -0.049 & 2.405 & 2.130 \\
\hline
\end{tabular}

Table 5: Measurements for the War Memorial.

\begin{tabular}{|l|c|c|c|}
\hline Dataset & GNSS & Barometer & Both \\
\hline Limestone Blocks & $-100 \%$ & $-17.9 \%$ & $-28.9 \%$ \\
War Memorial & $-102 \%$ & $8.65 \%$ & $3.76 \%$ \\
\hline
\end{tabular}

Table 6: Percentage error for each set of sensors.

\section{CONCLUSION}

In this paper we present a sensor fusion method to fuse accelerometer, barometer and GNSS data using Non-Parametric Belief Propagation. Once a device is calibrated this method requires no setup, control points or extra work on behalf of the person capturing the data. All of the analysis and scaling is performed without extra input or physical measurement on the actual objects being reconstructed. This is potentially useful in several cases where access is difficult or impossible or it is not possible to touch or actively scan the objects.

While these results are preliminary the data is less than ideal suggesting that there is potential to get useful results even in the presence of significant outliers. For example, despite the very large errors in the GNSS data we are still able to achieve results that have much less error that the input data.

\subsection{Future Work}

While this implementation of sensor fusion shows improvement over just using the raw sensor data there is scope for improvement in several ways. For example, when there are other sensors available it would be useful to attempt to automatically calibrate sensors such as the barometer using the additional data. This would remove the current method of returning to the start point which is not always possible in many situations.

In this implementation we utilise Photoscan to perform the photogrammetry component. This is not ideal for several reasons. At the current time the algorithms used by Photoscan are not publicly known which limits transparency and understanding of what causes a particular error. Secondly, with the extra information available from multiple sensors as either constraints or as initialisation values implementation of our own or modification of an existing open source photogrammetry package would be ideal for making use of this data.

\section{REFERENCES}

Agisoft, LLC, 2016. Agisoft Photoscan. http://www . agisoft.com (16 Apr. 2016).

Ali, T., Dutta, P. and Boruah, H., 2012. A new combination rule for conflict problem of dempster-shafer evidence theory. International Journal of Energy, Information and Communications 3(1), pp. $35-40$.
Ayub, S., Bahraminasab, A. and Honary, B., 2012. A sensor fusion method for smart phone orientation estimation. In: Proceedings of the 13th Annual Post Graduate Symposium on the Convergence of Telecommunications, Networking and Broadcasting.

Crandall, D. J., Owens, A., Snavely, N. and Huttenlocher, D. P., 2013. Sfm with mrfs: Discrete-continuous optimization for largescale structure from motion. Pattern Analysis and Machine Intelligence, IEEE Transactions on 35(12), pp. 2841-2853.

Engineering Toolbox, 2016. Altitude above Sea Level and Air Pressure. http://www.engineeringtoolbox.com/ air-altitude-pressure-d_462.html (17 Apr. 2016).

Google, 2016a. Location - Android Developers. https://developer.android.com/reference/android/ location/Location.html\#getAccuracy() (12 Apr. 2016).

Google, 2016b. Sensors Overview - Android Developers. https://developer.android.com/guide/topics/ sensors/sensors_overview.html\#sensors-coords (12 Apr. 2016).

Ihler, A. T., Iii, J. and Willsky, A. S., 2005. Loopy belief propagation: Convergence and effects of message errors. In: Journal of Machine Learning Research, pp. 905-936.

Jøsang, A. and Pope, S., 2012. Dempsters rule as seen by little colored balls. Computational Intelligence 28(4), pp. 453-474.

Kerl, C., Sturm, J. and Cremers, D., 2013. Robust odometry estimation for rgb-d cameras. In: Robotics and Automation (ICRA), 2013 IEEE International Conference on, IEEE, pp. 3748-3754.

Klein, L. A., 1993. Sensor and data fusion concepts and applications. Society of Photo-Optical Instrumentation Engineers (SPIE), Bellingham, WA, USA, 131 p.

Koks, D. and Challa, S., 2003. An introduction to bayesian and dempster-shafer data fusion. Technical report DSTO-TR-1436, 35 p., http://dspace.dsto.defence.gov.au/dspace/ bitstream/1947/4316/5/DSTO-TR-1436\%20PR.pdf.

Lhuillier, M., 2012. Incremental fusion of structure-from-motion and gps using constrained bundle adjustments. Pattern Analysis and Machine Intelligence, IEEE Transactions on 34(12), pp. 2489-2495.

Murphy, K. P., Weiss, Y. and Jordan, M. I., 1999. Loopy belief propagation for approximate inference: An empirical study. In: Proceedings of the Fifteenth conference on Uncertainty in artificial intelligence, Morgan Kaufmann Publishers Inc., pp. 467475 .

Pearl, J., 1982. Reverend Bayes on inference engines: A distributed hierarchical approach. In: $A A A I$, pp. 133-136.

Sudderth, E., Ihler, A., Freeman, W. and Willsky, A., 2013. Nonparametric belief propagation. In: In IEEE Computer Society conference on computer vision and pattern recognition (CVPR), Vol. 1, pp. 605-612.

Tundo, M. D., Lemaire, E. and Baddour, N., 2013. Correcting smartphone orientation for accelerometer-based analysis. In: Medical Measurements and Applications Proceedings (MeMeA), 2013 IEEE International Symposium on, IEEE, pp. 58-62.

Yedidia, J. S., Freeman, W. T. and Weiss, Y., 2000. Generalized belief propagation. In: NIPS, Vol. 13, pp. 689-695.

Yedidia, J. S., Freeman, W. T. and Weiss, Y., 2003. Understanding belief propagation and its generalizations. Exploring artificial intelligence in the new millennium 8, pp. 236-239. 